\title{
Week 48 efficacy and central nervous system analysis of darunavir/ritonavir monotherapy versus darunavir/ritonavir with two nucleoside analogues
}

Antinori, Andrea ; Clarke, Amanda ; Svedhem-Johansson, Veronika ; Arribas, José R ; Arenas-Pinto, Alejandro ; Fehr, Jan ; Gerstoft, Jan ; Horban, Andrzej ; Clotet, Bonaventura ; Ripamonti, Diego ; Girard, Pierre-Marie ; Hill, Andrew M ; Moecklinghoff, Christiane

\begin{abstract}
BACKGROUND: In previous studies in virologically suppressed patients, protease inhibitor monotherapy has shown trends for more low-level elevations in HIV-1 RNA compared with triple therapy, but no increase in the risk of drug resistance. METHODS: A total of 273 patients with HIV-1 RNA less than 50 copies/ml on first-line antiretrovirals switched to darunavir/ritonavir (DRV/r) 800/100 mg once daily, either as monotherapy $(\mathrm{n}=137)$ or as triple therapy with two nucleoside analogues $(\mathrm{n}=136)$. Treatment failure was defined as HIV-1 RNA levels 50 copies/ml or above, or discontinuation of study treatment by week 48 (FDA Snapshot algorithm). RESULTS: Patients were $83 \%$ male and $88 \%$ white, with mean age 42 years. In the primary efficacy analysis, HIV-1 RNA less than 50 copies/ml by week 48 [intention-to-treat (ITT)] was 118 of $137(86 \%)$ in the DRV/r monotherapy arm versus 129 of 136 $(95 \%)$ in the triple therapy arm (difference $=-8.7 \%, 95 \%$ confidence interval $-15.50,-1.80)$. In a post-hoc analysis, for patients with nadir CD4 cell count 200 cells/ l or above, rates of HIV-1 RNA suppression were 91 of $96(95 \%)$ in the DRV/r monotherapy arm and 100 of 106 (94\%) in the triple therapy arm. There was no difference in neurocognitive function or the risk of neuropsychiatric adverse events between $\mathrm{DRV} / \mathrm{r}$ monotherapy and triple therapy. Two patients in the monotherapy arm with CD4 nadir less than 200 cells/ l developed viraemia in both cerebrospinal fluid (CSF) and plasma, with one symptomatic case. CONCLUSIONS: In this study for patients with HIV-1 RNA less than 50 copies/ml at baseline, switching to $\mathrm{DRV} / \mathrm{r}$ monotherapy showed lower efficacy versus triple therapy at week 48 in the primary ITT switch equals failure analysis, with two cases of viraemia in the CSF in the protease inhibitor monotherapy arm.
\end{abstract}

DOI: https://doi.org/10.1097/QAD.0000000000000778

Posted at the Zurich Open Repository and Archive, University of Zurich

ZORA URL: https://doi.org/10.5167/uzh-114679

Journal Article

Published Version

Originally published at:

Antinori, Andrea; Clarke, Amanda; Svedhem-Johansson, Veronika; Arribas, José R; Arenas-Pinto, Alejandro; Fehr, Jan; Gerstoft, Jan; Horban, Andrzej; Clotet, Bonaventura; Ripamonti, Diego; Girard, Pierre-Marie; Hill, Andrew M; Moecklinghoff, Christiane (2015). Week 48 efficacy and central nervous system analysis of darunavir/ritonavir monotherapy versus darunavir/ritonavir with two nucleoside analogues. AIDS, 29(14):1811-1820.

DOI: https://doi.org/10.1097/QAD.0000000000000778 


\title{
Week 48 efficacy and central nervous system analysis of darunavir/ritonavir monotherapy versus darunavir/ ritonavir with two nucleoside analogues
}

\author{
Andrea Antinori ${ }^{\mathrm{a}}$, Amanda Clarke ${ }^{\mathrm{b}}$, Veronika Svedhem-Johansson ${ }^{\mathrm{c}}$, \\ José R. Arribass ${ }^{\mathrm{d}}$, Alejandro Arenas-Pinto ${ }^{\mathrm{e}}$, Jan Fehr ${ }^{\mathrm{f}}$, Jan Gerstoft ${ }^{\mathrm{g}}$, \\ Andrzej Horban ${ }^{\text {h }}$, Bonaventura Clotet', Diego Ripamonti ${ }^{j}$, \\ Pierre-Marie Girard ${ }^{\mathrm{k}}$, Andrew M. Hill' and Christiane Moecklinghoff ${ }^{\mathrm{m}}$
}

\begin{abstract}
Background: In previous studies in virologically suppressed patients, protease inhibitor monotherapy has shown trends for more low-level elevations in HIV-1 RNA compared with triple therapy, but no increase in the risk of drug resistance.

Methods: A total of 273 patients with HIV-1 RNA less than 50 copies/ml on first-line antiretrovirals switched to darunavir/ritonavir (DRV/r) 800/100 mg once daily, either as monotherapy ( $n=137)$ or as triple therapy with two nucleoside analogues $(n=136)$. Treatment failure was defined as HIV-1 RNA levels 50 copies/ml or above, or discontinuation of study treatment by week 48 (FDA Snapshot algorithm).
\end{abstract}

Results: Patients were $83 \%$ male and $88 \%$ white, with mean age 42 years. In the primary efficacy analysis, HIV-1 RNA less than 50 copies/ml by week 48 [intention-totreat (ITT)] was 118 of 137 (86\%) in the DRV/r monotherapy arm versus 129 of 136 $(95 \%)$ in the triple therapy arm (difference $=-8.7 \%, 95 \%$ confidence interval -15.50 , -1.80 ). In a post-hoc analysis, for patients with nadir $\mathrm{CD} 4^{+}$cell count 200 cells $/ \mu$ l or above, rates of HIV-1 RNA suppression were 91 of $96(95 \%)$ in the DRV/r monotherapy arm and 100 of $106(94 \%)$ in the triple therapy arm. There was no difference in neurocognitive function or the risk of neuropsychiatric adverse events between DRV/r monotherapy and triple therapy. Two patients in the monotherapy arm with $\mathrm{CD} 4^{+}$nadir less than 200 cells/ $\mu$ l developed viraemia in both cerebrospinal fluid (CSF) and plasma, with one symptomatic case.

Conclusions: In this study for patients with HIV-1 RNA less than 50 copies/ml at baseline, switching to DRV/r monotherapy showed lower efficacy versus triple therapy at week 48 in the primary ITT switch equals failure analysis, with two cases of viraemia in the CSF in the protease inhibitor monotherapy arm.

Copyright (c) 2015 Wolters Kluwer Health, Inc. All rights reserved.

AIDS 2015, 29:1811-1820

Keywords: darunavir, HIV clinical trials, nucleoside analogues, protease inhibitor monotherapy, ritonavir

\footnotetext{
${ }^{a}$ National Institute for Infectious Diseases, L. Spallanzani IRCCS, Rome, Italy, ${ }^{b}$ Brighton and Sussex University Hospitals, Brighton, UK, ' Karolinska University, Sjukhuset, Sweden, ${ }^{\mathrm{d} H}$ Hospital Universitario La Paz, IdiPAZ, Madrid, Spain, ${ }^{\mathrm{M}}$ Medical Research Council, HIV Clinical Trials Unit, London, UK, 'Division of Infectious Diseases and Hospital Epidemiology, University Hospital Zürich, University of Zürich, Zürich, Switzerland, ' ${ }^{\mathrm{g}}$ Copenhagen University Hospital, Copenhagen, Denmark, ${ }^{\mathrm{h}}$ Warsaw Medical University, Infectious Diseases, Warsaw, Poland, 'University Hospital Germans Trias I Pujol, irsiCaixa, Badalona, Spain, 'Infectious Diseases, Ospedale Papa Giovanni XXIII, Bergamo, Italy, kDepartment of Infectious and Tropical Diseases, Hôpital Saint-Antoine, AP-HP, and INSERM UMR S 1136, Paris, France, 'University of Liverpool, Liverpool, UK, and ${ }^{\mathrm{m} J a n s s e n, ~ E M E A, ~}$ Neuss, Germany.

Correspondence to Dr Andrea Antinori, National Institute for Infectious Diseases, L. Spallanzani IRCCS, Rome, Italy.

Tel: +390655170 456; e-mail: andrea.antinori@inmi.it

Received: 9 March 2015; revised: 27 April 2015; accepted: 11 June 2015.
}

DOI:10.1097/QAD.0000000000000778

ISSN 0269-9370 Copyright (C) 2015 Wolters Kluwer Health, Inc. All rights reserved. 


\section{Introduction}

Despite the success of protease inhibitor-based treatment [1], long-term use of combination antiretroviral therapy may be impaired by a variety of factors, including poor adherence, pill burden, and toxicity, and presents a high financial cost to healthcare systems [2,3]. Protease inhibitor monotherapy has been explored as a maintenance strategy in patients virologically suppressed on antiretrovirals to reduce the impact of these issues while aiming to maintain HIV-1 RNA suppression. Two metaanalyses have shown that patients switching to protease inhibitor monotherapy have a lower chance of maintaining HIV-1 RNA suppression [4,5]. However, in both analyses, there was similar efficacy between protease inhibitor monotherapy and triple therapy when reintroduction of the nucleoside/nucleotide reverse transcriptase inhibitor (NRTI) backbone was permitted. Furthermore, when used as monotherapy, there is a theoretical concern that protease inhibitors may not sufficiently penetrate into the central nervous system (CNS) and the protease inhibitor concentration observed may not be sufficient to suppress HIV-1 in this compartment [6]. If this is the case, the ongoing replication of HIV-1 in the cerebrospinal fluid (CSF) could lead to a variety of neuropsychiatric disorders and neurocognitive impairment [6,7]. Although evidence generally indicates that this is not the case, the concern is a potential barrier to a widespread protease inhibitor monotherapy approach and has not been evaluated systematically in most previous trials of protease inhibitor monotherapy $[6,7]$.

Darunavir boosted with ritonavir (DRV/r) has shown high levels of efficacy in treatment-naive and treatmentexperienced patients when co-administered with other antiretrovirals [8,9]. Moreover, darunavir has a high genetic barrier to the development of resistance, a long half-life compared with other protease inhibitors, and a favorable toxicity profile, making it an ideal candidate for use in monotherapy $[10,11]$.

The efficacy of DRV/r monotherapy versus $\mathrm{DRV} / \mathrm{r}$ plus two nucleoside analogues has been evaluated in patients suppressed on their previous regimen and without prior failure $[12,13]$. In both the MONET and MONOI studies, a trend for more low-level elevations in plasma HIV-1 RNA in the monotherapy arm compared with the triple therapy arm was observed. The long-term results of both studies suggest noninferior efficacy of $\mathrm{DRV} / \mathrm{r}$ monotherapy compared with triple therapy provided that NRTI backbone can be re-introduced as necessary [1416]. In the ongoing 5-year PIVOT study, protease inhibitor monotherapy was noninferior to triple therapy in preserving future treatment options, however was associated with a higher occurrence of viral rebound [17]. In these trials, the risk of drug resistance was minimal, and both regimens resulted in a similar number of adverse events. Together, these results suggest that $\mathrm{DRV} / \mathrm{r}$ monotherapy could represent a durable and efficacious option when used as a tailored treatment option for patients fully suppressed on triple therapy.

Monotherapy with a boosted protease inhibitor is currently not standard-of-care, but has been included in some treatment guidelines, such as the European AIDS Clinical Society (EACS) guidelines, as a treatment option in suppressed patients without prior failure to protease inhibitors [18]. This recommendation is currently omitted from other guidelines [19-21].

The aim of the PROTEA trial was to assess the noninferiority of monotherapy therapy with $\mathrm{DRV} / \mathrm{r}$ versus DRV/r triple therapy, and to evaluate the safety and tolerability of the regimen. Neurocognitive function was assessed throughout the study to determine whether protease inhibitor monotherapy confers a higher risk of neurocognitive impairment. Furthermore, lumbar punctures were performed in a subset of participants to assess viral replication in the CNS. Such comprehensive CNS evaluations were not performed in the previous studies of $\mathrm{DRV} / \mathrm{r}$ monotherapy.

\section{Methods}

PROTEA was a 96-week, randomized, open-label phase $3 \mathrm{~b}$ study, with study centers in 13 European countries and Israel. The trial recruited patients who had HIV-1 RNA suppression below 50 copies $/ \mathrm{ml}$ on their first-line antiretroviral regimen for the 48 weeks prior to screening. Key exclusion criteria included patients with a $\mathrm{CD} 4^{+}$ count $100 \mathrm{cells} / \mu \mathrm{l}$ or less at the start of antiretroviral therapy (nadir) and $200 \mathrm{cells} / \mu \mathrm{l}$ or less at screening, history of virological failure or prior protease inhibitor mutations.

During the study, the protocol was amended to allow intensification with nucleoside analogues for any patient in the monotherapy arm who had entered the trial with a $\mathrm{CD}^{+}$nadir below $100 \mathrm{cells} / \mu \mathrm{l}$. This amendment was introduced because patients with $\mathrm{CD}^{+}$nadir below 100 cells $/ \mu 1$ were protocol violators and should not be receiving protease inhibitor monotherapy.

Subsequent to screening, patients entered a 4-week runin period (baseline 1) in which all patients received DRV/ r 800/100 mg once daily with their current two NRTIs. Patients were then randomized (baseline 2) 1:1 to receive either DRV/r 800/100 mg once daily as monotherapy (monotherapy arm) or in combination with two NRTIs (triple therapy arm). The investigator-selected nucleoside analogues were either tenofovir, abacavir or zidovudine in combination with either lamivudine or emtricitabine. 
Randomization was stratified by HCV antibody status (anti-HCV negative or positive).

\section{Efficacy and safety assessments}

Patients attended study visits at screening, baselines 1 and 2 and then at weeks 4, 24 and thereafter every 24 weeks until the end of treatment (week 96). Evaluations for efficacy and safety were carried out at every study visit according to local standard-of-care; neurocognitive function was also assessed. Evaluation of plasma HIV-1 RNA levels was determined using the Abbott RealTime HIV-1 assay (lower quantification limit 40 copies/ml). Additionally, genotyping was carried out for all patients with two consecutive HIV-1 RNA levels more than 400 copies $/ \mathrm{ml}$. In a subgroup of patients, lumbar punctures were performed at baseline and again at week 48 to assess HIV-1 RNA levels in the CSF.

Switch of nucleoside analogues in the triple therapy arm was allowed at any visit in the event of suspected toxicity. Any subject in the monotherapy arm with virologic failure could have their treatment intensified with two nucleoside analogues, provided that major protease inhibitor mutations had not developed.

The Division of AIDS (DAIDS) grading tables were used to define clinical and laboratory abnormalities. An independent Data and Safety Monitoring Board (DSMB) was established to monitor data on an ongoing basis to ensure the continuing safety of the patients enrolled into the study.

Neurocognitive function was assessed using a series of neuropsychological tests: the revised Hopkins Verbal Learning Test (HVLT-r), excluding the retention and recognition tests, the Color Trail Test and the Grooved Pegboard Test [22-24]. The most common neurocognitive impairments seen in HIV-infected individuals are those that affect frontal subcortical functions, and as such, these tests were chosen to detect such changes [25].

Patients participating in the study provided informed consent prior to any study procedures. Approval from independent ethics committees and health authorities was obtained before initiating the study.

\section{Statistical methods}

The primary endpoint for the study was confirmed plasma HIV-1 RNA below 50 copies/ml at week 48 (FDA snapshot algorithm). All patients who discontinued or switched randomized study medication were considered nonresponders, inclusive of patients intensifying treatment with NRTIs in the monotherapy arm. Patients with missing HIV-1 RNA results at the 48-week visit were classified as having HIV-1 RNA more than 50 copies $/ \mathrm{ml}$.
Assuming a response rate of $90 \%, 130$ patients were targeted for recruitment in each arm to establish noninferiority a one-sided significance level of $2.5 \%$, $80 \%$ power, a noninferiority margin of $-12 \%$, and allowing for a maximum of $10 \%$ of patients to be excluded from the Per Protocol population. The primary population was the intention-to-treat (ITT) population; the Per Protocol population was analyzed to investigate the impact of exclusion of major protocol violations.

For the primary analysis, a logistic regression model including treatment arm and the stratification factor (anti$\mathrm{HCV}$ positive or negative at screening) was used to estimate the difference in virologic response rate between treatment arms, with corresponding 95\% confidence interval (CI). Sensitivity analyses incorporating additional covariates were conducted to examine the impact of differences in baseline factors.

In the secondary, switch-included analysis, all patients who discontinued randomized medications were followed up and their HIV-1 RNA levels at week 48 were included in the analysis, even if they had changed their antiretroviral treatment.

For the neurocognitive assessment, a total of five scores were determined and each standardized to give a normalized $z$ score using the manufacturers' normative data [22-24]. An overall score (NPZ-5) was derived by averaging the scores. The NPZ-5 score was dichotomized and considered abnormal if the standardized score was less than -1 , indicating below-average performance. Analysis of covariance (ANCOVA) was used to identify potential covariates affecting neurocognitive function and to provide an adjusted estimate for the NPZ-5 score at week 48 for each treatment group. The difference between arms was calculated and considered statistically significant if $P<0.05$.

\section{Central nervous system substudy}

A subgroup of subjects participated in the CNS substudy. These subjects underwent a lumbar puncture prior to baseline and again after 48 weeks of randomized treatment to assess CSF HIV-1 RNA levels. Lumbar puncture samples were sent to a central laboratory and CSF HIV-1 viral load was determined using the Abbott RealTime HIV-1 assay.

Patients were considered virologically suppressed in the CSF if HIV-1 RNA was below 50 copies $/ \mathrm{ml}$. In cases wherein CSF HIV-1 RNA elevations were observed, other indicators were investigated, including plasma HIV1 RNA, other disease markers and presentation of clinical symptoms. In addition, all CSF samples were assessed for other key disease markers, including albumin, neopterin and lymphocyte counts. 


\section{Results}

Figure 1 shows the overall patient disposition. In total, 325 patients were screened across 14 countries. Of these, 282 entered the 4-week run-in period and 273 eligible patients were randomized and treated with $\mathrm{DRV} / \mathrm{r}$ (137 in the monotherapy arm and 136 in the triple therapy; ITT population). One further patient was randomized, but excluded from the ITT population, as the patient did not take study medication in the treatment phase. Twenty-seven patients were excluded from the PerProtocol population (14 in the monotherapy arm and 13 in the triple therapy arm). The main reasons for exclusion were low nadir or screening $\mathrm{CD} 4^{+}$cell count, history of virologic failure, previous mono or dual antiretroviral therapy or a history of severe depression. There were eight patients randomized to the study despite having a nadir $\mathrm{CD}^{+}{ }^{+}$cell count lower than 100 cells/ $\mu \mathrm{l}$ : five in the monotherapy arm and three in the triple therapy arm. These patients were protocol violators, and were all excluded from the per protocol population. Following the protocol amendment, three of the five patients in the monotherapy arm with $\mathrm{CD} 4^{+}$nadirs below 100 cells $/ \mu 1$ were intensified with nucleoside analogues despite having HIV-1 RNA below 50 copies/ml. These three patients were included in the ITT analyses, but classified as treatment failures. In addition to the eight patients with $\mathrm{CD}^{+}$nadir below 100 cells $/ \mu 1$, there were 63 with $\mathrm{CD}^{+}{ }^{+}$nadir between 100 and 200 cells/ $\mu$, 36 in the monotherapy arm and 27 in the triple therapy arm.

Baseline characteristics by treatment group are shown in Table 1. Overall, $83 \%$ of patients were male, $87 \%$ were white, and the mean age was 42 years. The average duration of HIV-1 infection was 7.7 years and time since first antiretroviral therapy was 5.5 years. Twenty-six (10\%) patients were $\mathrm{HCV}$ antibody-positive at screening (13 patients in each arm). However, all 26 patients had undetectable levels of HCV RNA by PCR. In the triple therapy arm, concomitant NRTIs were tenofovir + emtricitabine for $71 \%$ of patients, and abacavir + lamivudine for $24 \%$ of patients; $6 \%$ were taking other combinations of nucleoside analogues.

\section{Efficacy}

In the primary ITT, switch equals failure analysis, 118 of 137 (86.1\%) in the DRV/r monotherapy arm compared with 129 of $136(94.9 \%)$ in the triple therapy arm had HIV-1 RNA below 50 copies/ $\mathrm{ml}$ at week 48 using the FDA snapshot algorithm (Fig. 2a). The difference of predicted response rates fell below the noninferiority margin of $-12 \%$ [difference $(\Delta) ;-8.7 \%, 95 \%$ confidence interval $(\mathrm{CI}),-15.50$ to -1.80$]$. As planned in the protocol, the primary analysis was repeated adjusting for differences in baseline disease characteristics; the final model included treatment group, HCV status, nadir $\mathrm{CD} 4^{+}$cell count and previous protease inhibitor use. In this analysis, noninferiority of monotherapy to triple therapy was shown $(\Delta-5.8 \%$; 95\% CI, $-11.51,-0.14)$ according to the predetermined noninferiority margin; however, the difference remained significantly inferior statistically. In the per protocol, switch equals failure analysis, 110 of $123(89.4 \%)$ in the monotherapy arm and 118 of $123(95.9 \%)$ in the triple therapy arm had HIV-1 RNA below 50 copies/ml at week 48 (Fig. 2a).

In the ITT, switch-included analysis, $92.0 \%$ of the DRV/r monotherapy arm and $96.3 \%$ of the triple therapy arm had HIV-1 RNA below 50 copies/ml at week 48 ; in the

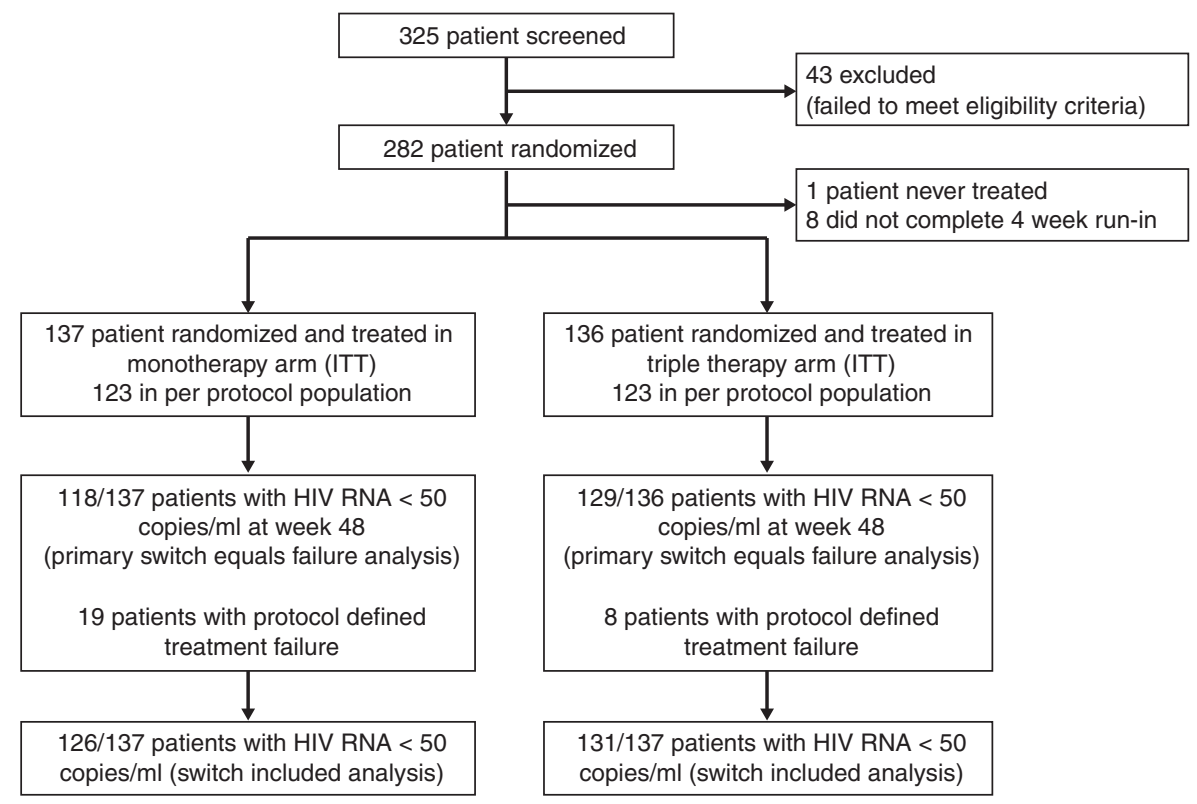

Fig. 1. Patient flowchart. 
Table 1. Baseline characteristics by treatment arm (ITT population).

\begin{tabular}{|c|c|c|c|}
\hline & Monotherapy $(n=137)$ & Triple therapy $(n=136)$ & Overall $(n=273)$ \\
\hline Mean age (years, $\pm \mathrm{SD}$ ) & $44.6( \pm 11.2)$ & $43.1( \pm 10.4)$ & $43.9( \pm 10.8)$ \\
\hline Sex (no. \% male) & $111(81)$ & $115(85)$ & $226(83)$ \\
\hline Race (no. \% White) & $119(87)$ & $120(88)$ & $239(88)$ \\
\hline Mean weight $(\mathrm{kg}, \pm \mathrm{SD})$ & $76.2( \pm 15.9)$ & $75.9( \pm 13.1)$ & $76.0( \pm 14.5)$ \\
\hline Mean BMI $\left(\mathrm{kg} / \mathrm{m}^{2}, \pm \mathrm{SD}\right)$ & $25.0( \pm 4.6)$ & $24.8( \pm 4.0)$ & $24.9( \pm 4.3)$ \\
\hline Known duration of HIV-1 infection (years, \pm SD) & $8.1( \pm 5.0)$ & $7.2( \pm 5.0)$ & $7.7( \pm 5.0)$ \\
\hline Duration of antiretroviral treatment (years) & $5.7( \pm 4.1)$ & $5.3( \pm 4.1)$ & $5.5( \pm 4.1)$ \\
\hline HCV antibody positive (no. \%) & $13(9)$ & $13(10)$ & $26(10)$ \\
\hline \multicolumn{4}{|l|}{ Baseline HIV-1 RNA category (no. \%) } \\
\hline$<50$ copies/ml & $137(100)$ & $133(98)$ & $270(99)$ \\
\hline $50-<400$ copies $/ \mathrm{ml}$ & 0 & $1(1)$ & $1(<1)$ \\
\hline$\geq 400$ copies $/ \mathrm{ml}$ & 0 & $2(1)$ & $2(1)$ \\
\hline \multicolumn{4}{|l|}{ Baseline CD $4^{+}$cell count category (no. \%) } \\
\hline$<200$ cells/ $\mu$ l & 0 & $1(1)$ & $1(<1)$ \\
\hline $200-<350$ cells $/ \mu l$ & $14(10)$ & $8(6)$ & $22(8)$ \\
\hline$\geq 350 \mathrm{cells} / \mu \mathrm{l}$ & $123(90)$ & $127(93)$ & $250(92)$ \\
\hline \multicolumn{4}{|l|}{ Nadir CD4 ${ }^{+}$cell count (no. \%) } \\
\hline$<100$ cells $/ \mu \mathrm{l}$ & $5(4)$ & $3(2)$ & $8(3)$ \\
\hline $100-<200$ cells $/ \mu l$ & $36(26)$ & $27(20)$ & $63(23)$ \\
\hline$\geq 200 \mathrm{cells} / \mu \mathrm{l}$ & $96(70)$ & $106(78)$ & $202(74)$ \\
\hline \multicolumn{4}{|l|}{ Clinical stage (CDC) of HIV-1 infection } \\
\hline Category A & $96(70)$ & $110(81)$ & $206(75)$ \\
\hline Category B & $27(20)$ & $17(13)$ & $44(16)$ \\
\hline Category C & $14(10)$ & $9(7)$ & $23(8)$ \\
\hline On first NRTI combination (no. \%) & $79(58)$ & $96(71)$ & $175(64)$ \\
\hline Protease inhibitor naive at screening (no. \%) & $30(22)$ & $24(18)$ & $54(20)$ \\
\hline Protease inhibitor treatment at screening (no. \%) & $95(69)$ & $103(76)$ & $198(73)$ \\
\hline NNRTI treatment at screening (no. \%) & $35(26)$ & $31(23)$ & $66(24)$ \\
\hline Included in CNS substudy (no. \%) & $37(27)$ & $34(25)$ & $71(26)$ \\
\hline Evaluable CSF sample at baseline (no. $\%{ }^{a}$ ) & $28(76)$ & $29(85)$ & $57(80)$ \\
\hline Evaluable CSF sample at week $48\left(\right.$ no. $\left.\%^{\mathrm{a}}\right)$ & $21(57)$ & $19(56)$ & $40(56)$ \\
\hline
\end{tabular}

Percentages may not add to $100 \%$ because of rounding. CDC, Centers for Disease Control and Prevention; CNS, central nervous system; CSF, cerebrospinal fluid; HCV, hepatitis C virus; NNRTI, nonnucleoside reverse transcriptase inhibitor; NRTI, nucleoside reverse transcriptase inhibitor; RNA, ribonucleic acid; SD, standard deviation.

${ }^{\text {a }}$ Shows percentage of subjects included in CNS substudy.

per protocol, switch-included analysis, proportions were 91.9\% and $96.7 \%$ for the DRV/r monotherapy and triple therapy arms, respectively (Fig. 2b). In all analyses of the switch-included population, noninferiority of DRV/r monotherapy to triple therapy was shown.

In the multiple regression model, low nadir $\mathrm{CD} 4^{+}$cell counts and previous use of protease inhibitors were the main predictors of treatment failure $(P=0.005$ and $P=0.004$, respectively). More detailed analysis showed that a threshold for nadir $\mathrm{CD}^{+}{ }^{+}$of 200 cells $/ \mu 1$ was the best predictor of treatment failure. In a post-hoc analysis for patients with nadir $\mathrm{CD}^{+}{ }^{+}$count below 200 cells/ $\mu \mathrm{l}$, 27 of $41(65.9 \%)$ in the monotherapy arm and 29 of 30 $(96.7 \%)$ in the triple therapy arm were virologically suppressed at week 48 . For those with a nadir count above 200 cells/ $\mu$ l, the proportion with HIV-1 RNA below 50 copies/ml at week 48 was 91 of $96(94.8 \%)$ in the $\mathrm{DRV} / \mathrm{r}$ monotherapy arm and 100 of 106 (94.3\%) in the triple therapy arm (Fig. 2c).

Genotypic data were available for the three patients who experienced confirmed HIV-1 RNA elevations 400 copies/ $\mathrm{ml}$ or above (two patients in monotherapy arm and one patient from triple therapy arm). No treatment-emergent primary protease inhibitor mutations were detected.

\section{Safety}

Summary safety results are shown in Table 2. Overall, $66 \%$ of the patients had at least one adverse event up to week 48; the most common adverse events were infections or infestations (32\%) and gastrointestinal $(16 \%)$. Fourteen $(5 \%)$ patients reported at least one serious adverse event (nine in the monotherapy arm and five in the triple therapy arm). Grade 2-4 adverse events considered treatment-related were more common in the monotherapy arm $(n=12 ; 9 \%)$ than the triple therapy $\operatorname{arm}(n=2 ; 1 \%)$. In the monotherapy arm, these were mainly gastrointestinal adverse events and rises in cholesterol after discontinuation of tenofovir.

By week 48, five patients (4\%) in the monotherapy arm and one patient (1\%) in the triple therapy arm permanently discontinued darunavir as a result of adverse events; additionally, one patient in the triple therapy arm temporarily stopped darunavir treatment. One patient in the monotherapy arm died of a cardiac arrest and hyperkalemia deemed unrelated to study treatment by the trial investigator. 
(a)

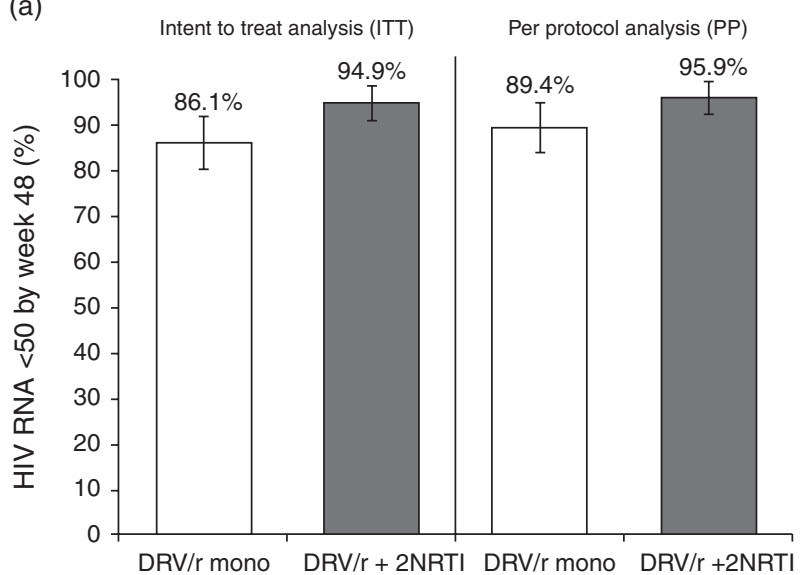

(b) Intent to treat analysis (ITT)

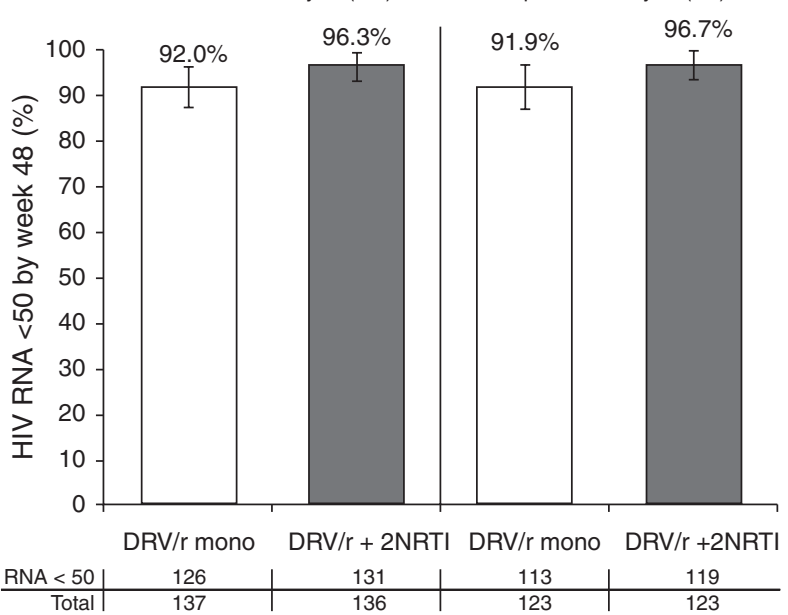

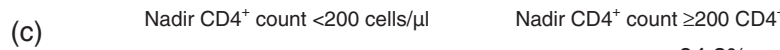

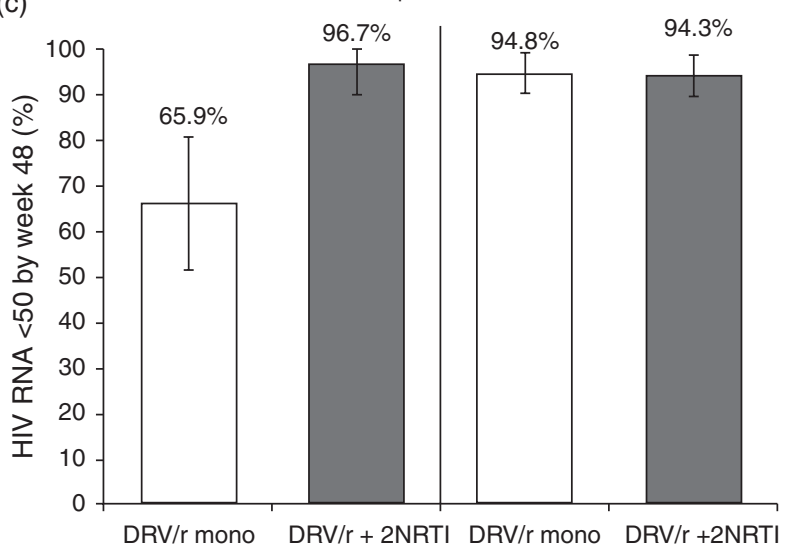

Fig. 2. HIV RNA suppression < 50 copies/ml at week 48 . (a) Primary efficacy analysis: HIV-1 RNA <50 copies/ml at week 48; FDA snapshot, switch equals failure. Shown are the percentage of patients suppressed (HIV-1 RNA $<50$ copies $/ \mathrm{ml}$ ) in the ITT and per protocol populations, where switches off randomization treatment were classified

Overall, 27 (10\%) patients experienced neurological adverse events (13 in the monotherapy arm and 14 in the triple therapy); the most common neurological adverse event was headache $(n=14 ; 5 \%)$. Nineteen $(7 \%)$ patients experienced psychiatric disorders (10 in the monotherapy arm and nine in the triple therapy), with depression $(n=6 ; 2 \%)$ and insomnia $(n=5 ; 2 \%)$, the most common. One patient in the monotherapy arm was hospitalized with HIV encephalomyelitis. This patient had a low nadir $\mathrm{CD}^{+}(17$ cells $/ \mu \mathrm{l})$, and at the time of hospitalization ( w week 24) had HIV-1 RNA detectable in the plasma and in the CSF (125 and 2500 copies/ml, respectively); the patient was re-suppressed and symptoms resolved after treatment intensification with nucleoside analogues including high-dose zidovudine (600 mg twice daily).

\section{Neurocognitive function}

Mean scores increased from screening to week 48 for all neurocognitive domains in both treatment groups; this is likely to be the result of a learning effect. There was no significant difference between the arms in the NPZ-5 score over time (Fig. 3). At week 48, the percentage of patients with an abnormal neurocognitive score was $12.2 \%$ for DRV/r monotherapy and $14.9 \%$ for triple therapy. This difference was not significant.

In ANCOVA analysis, sex, race, and baseline NPZ-5 score were found to have a highly significant effect on the week 48 NPZ-5 score $(P<0.0001)$. Alcohol consumption, smoking, history of cardiovascular events and age were also found to be significantly associated. Results were not affected by baseline HIV-1 RNA, baseline $\mathrm{CD}^{+}{ }^{+}$cell count or nadir $\mathrm{CD} 4^{+}$. After adjustment, mean

as treatment failure. In both populations, the difference between the $D R V / r$ monotherapy and $D R V / r$-based triple therapy arms did not meet the noninferiority margin of $-12 \%$ (ITT: $\Delta-8.7 \%, 95 \% \mathrm{Cl}-15.50,-1.80$; per protocol: $\Delta-6.5 \%, 95 \% \mathrm{Cl}-12.94,-0.04)$. DRV/r, darunavir/ritonavir; NRTI, nucleoside reverse transcriptase inhibitor. (b) Secondary efficacy analysis: HIV-1 RNA $<50$ copies/ml at week 48; FDA snapshot, switch included. Shown is the percentage of patients suppressed (HIV-1 RNA $<50$ copies/ml) in the ITT and per protocol populations in which switches off treatment, including re-intensification with NRTIs, were not considered treatment failures. In both populations, noninferiority of DRV/r monotherapy to DRV/r-based triple therapy was shown (ITT: $\Delta-4.3 \%, 95 \% \mathrm{Cl}-9.69,1.18$; per protocol: $\Delta-4.7 \%, 95 \% \mathrm{Cl}-10.49,1.00)$. (c) Posthoc analysis: HIV-1 RNA $<50$ copies/ml at week 48 by baseline nadir $\mathrm{CD} 4^{+}$cell count; FDA snapshot, switch equals failure. Shown are the percentage of patients suppressed (HIV-1 RNA $<50$ copies $/ \mathrm{ml}$ ) in the ITT switch=failure population by baseline nadir $\mathrm{CD}^{+}$cell count. For patients with nadir $\mathrm{CD}^{+}$cell count $\geq 200$ cells/ $\mu$ l, noninferiority of DRV/r monotherapy to DRV/r-based triple therapy was shown (Nadir $\mathrm{CD}^{+}<200: \Delta-30.8 \%, 95 \% \mathrm{Cl}-46.69,-14.94 ;$ Nadir $\left.\mathrm{CD}^{+} \geq 200: \Delta 0.5 \%, 95 \% \mathrm{Cl}-5.80,6.71\right)$. 
Table 2. Incidence of grade 1-4 adverse events, adverse events leading to permanent discontinuation of darunavir and incidence of nervous system and psychiatric adverse events by treatment arm.

\begin{tabular}{|c|c|c|c|}
\hline & Monotherapy $(n=137)$ & Triple therapy $(n=136)$ & Overall $(n=273)$ \\
\hline Any grade $1-4$ all-cause adverse events (no. $\%)^{a}$ & $96(70)$ & $83(61)$ & $179(66)$ \\
\hline Infections and infestations & $49(36)$ & $37(27)$ & $86(32)$ \\
\hline Gastrointestinal & $23(17)$ & $21(15)$ & $44(16)$ \\
\hline General/administration site & $18(13)$ & $19(14)$ & $37(14)$ \\
\hline Musculoskeletal and connective tissue & $20(15)$ & $15(11)$ & $35(13)$ \\
\hline Skin and subcutaneous tissue & $16(12)$ & $12(9)$ & $28(10)$ \\
\hline Nervous system & $13(9)$ & $14(10)$ & $27(10)$ \\
\hline Respiratory, thoracic, and mediastinal & $11(8)$ & $10(7)$ & $21(8)$ \\
\hline Investigations & $13(9)$ & $6(4)$ & $19(7)$ \\
\hline Metabolism and nutrition & $10(7)$ & $9(7)$ & $19(7)$ \\
\hline Psychiatric & $10(7)$ & $9(7)$ & $19(7)$ \\
\hline Any adverse event leading to permanent stop of DRV (no. \%) & $5(4)$ & $1(1)$ & $6(2)$ \\
\hline Gastrointestinal & $3(2)$ & 0 & $4(1)$ \\
\hline Metabolism and nutrition & $2(1)$ & 0 & $2(1)$ \\
\hline Cardiac disorders & $1(1)$ & 0 & $1(<1)$ \\
\hline Investigations & $1(1)$ & 0 & $1(<1)$ \\
\hline Nervous system & $1(1)$ & 0 & $1(<1)$ \\
\hline Pregnancy & 0 & $1(1)$ & $1(<1)$ \\
\hline Any serious adverse event (no. \%) & $9(7)$ & $5(4)$ & $14(5)$ \\
\hline Any grade $2-4$ treatment-related adverse events (no. \%) & $12(9)$ & $2(1)$ & $14(5)$ \\
\hline \multicolumn{4}{|l|}{ Grade $1-4$ nervous system and psychiatric adverse events (no. \%) } \\
\hline Headache & $8(6)$ & $6(4)$ & $14(5)$ \\
\hline Depression & $3(2)$ & $3(2)$ & $6(2)$ \\
\hline Insomnia & $3(2)$ & $2(1)$ & $5(2)$ \\
\hline Anxiety & $2(1)$ & $1(1)$ & $3(1)$ \\
\hline \multicolumn{4}{|l|}{ Serious nervous system and psychiatric adverse events (no. \%) } \\
\hline Encephalomyelitis & $1(<1)$ & 0 & $1(<1)$ \\
\hline Ischaemic stroke & $1(<1)$ & 0 & $1(<1)$ \\
\hline Substance abuse & 0 & $1(<1)$ & $1(<1)$ \\
\hline
\end{tabular}

DRV, darunavir.

${ }^{a}$ Affecting more than $5 \%$ of patients overall.

${ }^{\mathrm{b}}$ Affecting $\geq 3$ patients overall.

NPZ-5 score at week 48 was similar across the two groups (difference $0.007 ; 95 \% \mathrm{CI}-0.128,0.141 ; P=0.923$ ).

\section{Central nervous system substudy}

In total, 71 patients were included in the CNS substudy (37 monotherapy and 34 triple therapy; 26\% of total participants). The baseline characteristics of this substudy were similar to those in the overall trial. Of participants in the substudy, $80 \%$ and $56 \%$ had evaluable CSF data at baseline and week 48 , respectively (Table 1 ).

At baseline, all patients with measurable samples were virologically suppressed in the CSF (HIV-1 RNA $<50$ copies $/ \mathrm{ml}$ ). By week 48 , one patient had elevated CSF HIV-1 RNA (654 copies/ml); all other patients remained suppressed. The patient experiencing CSF elevations was in the monotherapy arm and was asymptomatic, however had a concurrent rise in plasma HIV-1 RNA ( 77 copies $/ \mathrm{ml}$ ) and a low $\mathrm{CD}^{+}{ }^{+}$nadir (166 cells/ $\mu \mathrm{l})$. The patient had neopterin levels above the reference range at baseline, which further increased to week 48 (7.13 and $11.15 \mathrm{nmol} / 1$ respectively; reference range $0.00-5.50 \mathrm{nmol} / \mathrm{l})$.

Overall, in the monotherapy arm, mean (standard deviation) CSF neopterin concentration rose from 4.8 (2.1) $\mathrm{nmol} / \mathrm{l}$ at baseline, to $6.2(4.3) \mathrm{nmol} / \mathrm{l}$ at week 48 . In the triple therapy arm, mean neopterin level remained constant over time [4.8 (1.3) at baseline vs. 4.1 (1.2) at week 48]. Mean CSF albumin was in the normal range $(100-300 \mathrm{mg} / \mathrm{l})$ at week 48 for both treatment groups.

\section{Discussion}

In the primary 48-week analysis of the PROTEA trial, using a switch equals failure endpoint, rates of HIV-1 RNA suppression below 50 copies $/ \mathrm{ml}$ were 118 of 137 (86.1\%) in the DRV/r monotherapy arm compared with 129 of $136(94.9 \%)$ in the triple therapy arm. In a secondary 'switch-included' analysis, which classified HIV-1 RNA suppression after re-introduction of nucleoside analogues as treatment success, 126 of 137 (92.0\%) in the DRV/r monotherapy arm and 131 of 137 $(96.3 \%)$ in the triple therapy arm showed virological suppression at week 48 .

These results are similar to the final 144-week analysis of the MONET trial and the 96-week analysis of the MONOI, which both had a similar design. In the MONET and MONOI trials, DRV/r monotherapy displayed lower rates of HIV-1 RNA suppression than triple therapy in the primary switch equals failure analysis, 


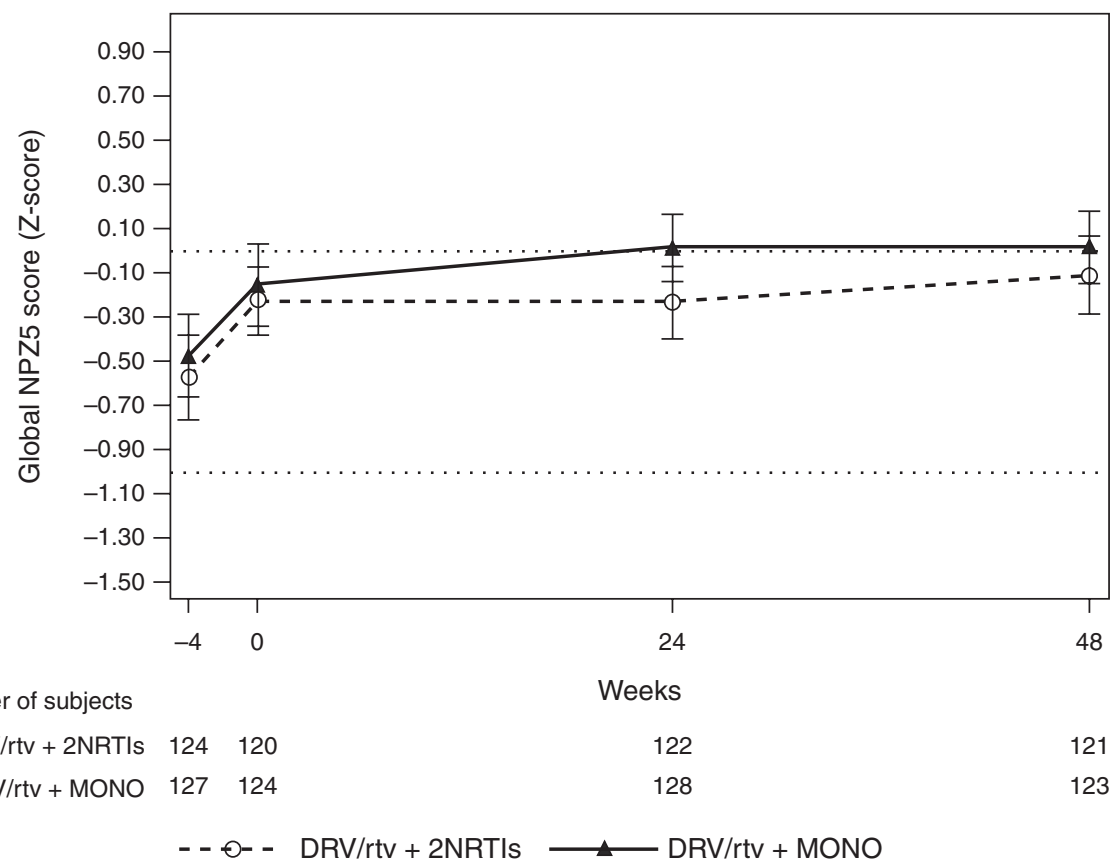

Fig. 3. Neurocognitive function. Mean $( \pm 95 \% \mathrm{Cl})$ global neurocognitive $(\mathrm{NPZ}-5)$ score over time.

but similar rates of HIV-1 RNA suppression in the switch-included analysis $[13,15]$.

In the present study, a low nadir $\mathrm{CD}^{+}$count $(<200$ cells $/ \mu l)$ was highly predictive of treatment failure in the monotherapy arm. Patients with a nadir $\mathrm{CD}^{+}$cell count over 200 cells $/ \mu l$ experienced similar response rates irrespective of randomization arm. There were more patients with low $\mathrm{CD} 4{ }^{+}$nadir levels in the monotherapy arm $(30 \%)$ than the triple therapy arm $(22 \%)$, which may have influenced the overall outcome in the primary efficacy analysis. In studies of monotherapy with LPV/r, a low nadir $\mathrm{CD}^{+}$cell count has been found to be predictive of treatment failure [26,27]. However, this is the first trial of DRV/r monotherapy in which nadir $\mathrm{CD} 4^{+}$cell count was found to be predictive of treatment response $[28,29]$. It should be acknowledged, however, that this was an exploratory subgroup analysis, and the original exclusion criterion from the PROTEA trial was a CD4 ${ }^{+}$nadir below 100 cells $/ \mu 1$; the 200 cells $/ \mu 1$ threshold should be evaluated in future studies of protease inhibitor monotherapy possibly through stratification prior to randomization.

Adverse events leading to the discontinuation of therapy were relatively rare in both arms. Despite this, DRV/r monotherapy was associated with a slightly higher rate of treatment-emergent adverse events. The most common side-effects in both arms were gastrointestinal and infections and infestations and were generally mild to moderate in severity. Protease inhibitor use is widely associated with hepatic and gastrointestinal complications; however, the increased frequency in the monotherapy arm is problematic given that a reduction in side-effects is one of the main reasons for treatment simplification to monotherapy [30].

Regarding CNS outcomes, up to week 48 , no difference in neurocognitive function was observed between the two arms. Furthermore, the incidence of neurological and psychiatric adverse events was similar. These findings are generally in agreement with the results from other randomized protease inhibitor/ $\mathrm{r}$ monotherapy trials and cohorts [31-34]. A recent systematic review of 11 studies in 1267 patients showed no significant difference in risk of CNS adverse events between PI monotherapy versus triple therapy [31]. Similarly, in a literature review assessing neurocognitive performance, protease inhibitor/ $\mathrm{r}$ monotherapy was not associated with worse neurocognitive outcomes compared with triple therapy; however, the authors advised of the need for further evidence to confirm this finding [32]. Importantly, a low nadir $\mathrm{CD}^{+}$has been shown to be an independent predictor of neurocognitive impairment [35]. Thus, given the efficacy findings, it may be especially pertinent that protease inhibitor monotherapy is avoided in the subgroup of patients with a low nadir $\mathrm{CD}^{+}$cell count.

One patient in the DRV/r monotherapy arm developed HIV-encephalomyelitis requiring hospitalization; this patient was found to have substantially elevated HIV-1 RNA in the CSF. Although this could be a casual result of the switch to protease inhibitor/ $\mathrm{r}$ monotherapy, this cannot be confirmed, and could be the result of disease progression and consequent CNS inflammation. One further patient in the CNS substudy experienced 
asymptomatic viraemia in the CSF after switching to monotherapy. This patient had elevated neopterin at baseline and week 48. In a recent study, a correlation was observed between high neopterin levels and low-level HIV-1 RNA replication in the CNS despite plasma suppression on triple therapy [36]. This suggests that the CNS could have acted as a viral reservoir in this patient prior to inclusion in the study. In both cases, subjects experienced concurrent viraemia in the plasma and both had a nadir $\mathrm{CD}^{+}{ }^{+}$cell count below 200 cells $/ \mu 1$.

CSF HIV-1 RNA has been inadequately reported and, prior to this study, had only been assessed in two randomized protease inhibitor/ $\mathrm{r}$ monotherapy trials, MONOI and MOST [13,27]. In MONOI, CSF assessments were only taken in symptomatic patients. Two patients with CNS events in the protease inhibitor/r monotherapy arm were found to have detectable CSF HIV-1 RNA despite plasma suppression [13]. In the MOST study, the proportion with viremia in the CSF was significantly higher in patients on monotherapy compared with triple therapy [27]. Despite this, the MOST study had limited power to detect differences and the findings have since been contested [37]. In a recent small cross-sectional study, CSF viral suppression was similar in patients receiving long-term LPV/r monotherapy compared with $\mathrm{LPV} / \mathrm{r}$-containing triple therapy [38]. Of note, the findings of a recent study suggest low-level CSF replication can occur in some patients on triple therapy [36], and thus the effects of the CNS HIV-1 reservoir may not be restricted to patients receiving protease inhibitor/ r monotherapy.

In summary, DRV/r monotherapy may be suitable as a tailored treatment option for patients fully suppressed on therapy, where reintroduction of NRTIs can be performed as necessary. DRV/r monotherapy may be most appropriate for patients with nadir $\mathrm{CD} 4^{+}$cell count greater than 200 cells $/ \mathrm{mm}^{3}$; however, this threshold needs to be further investigated. CNS viral escape remains a concern and as such, future protease inhibitor monotherapy trials should be designed to incorporate CSF HIV-1 RNA evaluations.

\section{Acknowledgements}

This study was sponsored by Janssen.

\section{Conflicts of interest}

A.A. has received speaker's honoraria, educational, travel, and/or research grants from AbbVie, Bristol-Myers Squibb, Gilead Sciences, Janssen, Merck, and ViiV Healthcare. J.F. has received speaker's honoraria, educational, travel, and research grants and honoraria from AbbVie, Bristol-Myers Squibb, Gilead Sciences, Janssen, Merck, and ViiV Healthcare. M.F. has received speaker's honoraria, educational, travel, and research grants from AbbVie, Bristol-Myers Squibb, Gilead Sciences, and ViiV healthcare. J.A. has received advisory fees, speaker fees, and grant support from ViiV healthcare, Tibotec, Janssen, Abbvie, Bristol-Myers Squibb, Gilead Sciences, MSD, and Tobira. P.M.G. has received speaker's honoraria, educational, travel, and/or research grants from AbbVie, Bristol-Myers Squibb, Gilead Sciences, Janssen, Merck, and ViiV Healthcare. The other authors have no conflicts of interest.

Clinical trial identifier: NCT01448707

Trial registration number: NCT01448707

\section{References}

1. Panel on Antiretroviral Guidelines for Adults and Adolescents. Guidelines for the use of antiretroviral agents in HIV-1-infected adults and adolescents. Department of Health and Human Services. January 29, 2008; 1-128. http://www.aidsinfo.nih. gov/ContentFiles/AdultandAdolescentGL.pdf. [Accessed 15 February 2011]

2. Gazzard B, Moecklinghoff C, Hill A. New strategies for lowering the costs of antiretroviral treatment and care for people with HIV/AIDS in the United Kingdom. Clinicoecon Outcomes Res 2012; 4:193-200.

3. Gazzard B, Hill A, Anceau A. Cost-efficacy analysis of the MONET trial using UK antiretroviral drug prices. Appl Health Econ Health Policy 2011; 9:217-223.

4. Bierman WF, van Aftmael MA, Nijhuis M, Danner SA, Boucher CA. HIV monotherapy with ritonavir-boosted protease inhibitors: a systematic review. AIDS 2009; 23:279-291.

5. Mathis S, Khanlari B, Pulido F, Schechter M, Negredo E, Nelson $M$, et al. Effectiveness of protease inhibitor monotherapy versus combination antiretroviral maintenance therapy: a meta-analysis. PLOS ONE 2011; 6:e22003.

6. Perez-Valero I, Bayon C, Cambron I, Gonzalez A, Arribas JR. Protease inhibitor monotherapy and the CNS: peace of mind? J Antimicrob Chemother 2011; 66:1954-1962.

7. Powderly W, Hill A. Moecklinghoff. Is there a higher risk of CNS adverse events for PI monotherapy versus triple therapy? A review of results from randomized clinical trials. HIV Clin Trials 2014; 15:79-86.

8. Mills A, Nelson M, Jayaweera D, Ruxrungtham K, Cassetti I, Girard $\mathrm{P}$, et al. Once-daily darunavir/ritonavir vs lopinavirritonavir in treatment-naïve, HIV-1 infected patients: 96 week analysis of ARTEMIS. AIDS 2009; 23:1679-1688.

9. Madruga J, Berger D, McMurchie M, Suter F, Banhegyi D, Ruxrungtham K, et al. Efficacy and safety of darunavir-ritonavir compared with that of lopinavir-ritonavir at 48 weeks in treatment-experienced, HIV infected patients in TITAN: a randomized controlled phase III trial. Lancet 2007; 370: 49-58.

10. Boffito M, Miralles D, Hill A. Pharmacokinetics, efficacy and safety of darunavir/ritonavir $800 / 100 \mathrm{mg}$ once daily in treatment-naïve and experienced patients. HIV Clin Trials 2008; 9:418-427.

11. Noach, A.B.J., Paulus, G. Safety Assessment Document TMC114, Tibotec NV, December 2000.

12. Arribas J, Horban A, Gerstoft J, Fattkenheuer G, Nelson M, Clumeck N, et al. The MONET trial: darunavir/ritonavir with or without nucleoside analogues, for patients with HIV RNA below 50 copies/ml. AIDS 2010; 24:223-230.

13. Katlama C, Valentin MA, Algarte-Genin M, Duvivier C, Lambert-Niclot S, Girard PM, et al. Efficacy of darunavir/ritonavir maintenance monotherapy in patients with HIV-1 viral suppression: a randomized openlabel noninferiority trial, MONOI-ANRS 136. AIDS 2010; 24:2365-2374. 
14. Clumeck N, Rieger A, Banhegyi D, Schmidt W, Hill A, Van Delft $Y$, et al. 96 week results from the MONET trial: a randomized comparison of darunavir/ritonavir with versus without nucleoside analogues, for patients with HIV RNA $<50$ copies $/ \mathrm{mL}$ at baseline. I Antimicrob Chemother 2011; 66:1878-1885.

15. Arribas J, Clumeck N, Nelson M, Hill A, van Delft Y, Moecklinghoff $C$. The MONET trial: week 144 analysis of the efficacy of darunavir/ritonavir (DRV/r) monotherapy versus DRV/r plus two nucleoside reverse transcriptase inhibitors, for patients with viral load $<50$ HIV-1 RNA copies/mL at baseline. HIV Med 2012; 13:398-405.

16. Valantin MA, Lambert-Niclot S, Flandre P, Morand-Joubert L, Cabie A, Meynard JL, et al. Long-term efficacy of darunavir/ ritonavir monotherapy in patients with HIV-1 viral suppression: week 96 results from the MONOI ANRS 136 study. I Antimicrob Chemoter 2012; 67:691-695.

17. Patron N, Arenas-Pinto A, Dunn D, Stoehr W, Fleck S, Scott K, et al. The protease inhibitor monotherapy versus ongoing tripletherapy trial (PIVOT). BHIVA Conference, Liverpool, UK April 2014.

18. European AIDS Clinical Society (EACS) Guidelines. Clinical management and treatment of HIV infected adults in Europe, version 7.1 (2014). http://www.eacsociety.org/guidelines/eacsguidelines/eacs-guidelines.html [Accessed January 2015]

19. Thompson M, Aberg J, Cahn P, Montaner J, Rizzardini G, Telenti A, et al. Antiretroviral Treatment of Adult HIV Infection. 2010 Recommendations of the International AIDS Society-USA Panel. JAMA 2010; 304:321-333.

20. British HIV Association. British HIV Association guidelines for the treatment of HIV-1-positive adults with antiretroviral therapy 2012. HIV Med 2014; 15 (Suppl 1):1-85.

21. US Department of Health and Human Services Panel on Antiretroviral Guidelines or Adults and Adolescents. Guidelines for the use of antiretroviral agents in HIV-1-infected adults and adolescents (2014). http://aidsinfo.nih.gov/guidelines. [Accessed January 2015]

22. Brandt J, Benedict RHB. Hopkins Verbal Learning test-revised. Odessa, FL: Psychological Assessment Resources; 2001.

23. D'Elia LF, Satz P, Uchiyama CL, White T. Color Trails Test. Professional manual. Odessa, FL: Psychological Assessment Resources; 1996.

24. Lafayette Instrument. Grooved pegboard test user instructions. Lafayette, IL: Lafayette Instrument Company, Inc.; 2002.

25. Valcour V, Paul R, Chiao S, Wendelken LA, Miller B. Screening for cognitive impairment in human immunodeficiency virus. Clin Infect Dis 2011; 53:836-842.

26. Pulido F, Pérez-Valero I, Delgardo R, Arranz A, Pasquau J, Portilla J, et al. Risk factors for loss of virological suppression in patients receiving lopinavir/ritonavir monotherapy for maintenance of HIV suppression. Antivir Ther 2009; 14:195-201.
27. Gutmann C, Cusini A, Günthard HF, Fux C, Hirschel B, Decosterd LA, et al. Randomized controlled study demonstrating failure of LPV/r monotherapy in HIV: the role of compartment and CD4-nadir. AIDS 2010; 24:2347-2354.

28. Arribas J, Pulido F, Hill A, van Delft Y, Moecklinghoff C. Predictors of long-term HIV RNA suppression on darunavir/ ritonavir monotherapy in the MONET trial. J Int AIDS SOC 2012; 15 (Suppl 4):18354.

29. Lambert-Niclot S, Flandre P, Valantin MA, Peytavin G, Duvivier C, Haim-Boukobza S, et al. Factors associated with virological failure in HIV-1-infected patients receiving darunavir/ritonavir monotherapy. I Infect Dis 2011; 204:12111216.

30. Wu X, Li Y, Peng K, Zhou H. HIV protease inhibitors in gut barrier dysfunction and liver injury. Curr Opin Pharmacol 2014; 19:61-66.

31. Powderly W, Hill A, Moecklinghoff $C$. Is there a higher risk of CNS adverse events for PI monotherapy versus triple therapy? A review of results from randomized clinical trials. HIV Clin Trials 2014; 15:79-86.

32. Pérez-Valero I, Bayon C, Cambron I, Gonzalez A, Arribas IR. Protease inhibitor monotherapy and the CNS: peace of mind? J Antimicrob Chemother 2011; 66:1954-1962.

33. Pérez-Valero I, González-Baeza A, Estébanez M, Monge S, Montes-Ramírez ML, Bayón C, et al. A prospective cohort study of neurocognitive function in aviremic HIV-infected patients treated with 1 or 3 antiretrovirals. Clin Infect Dis 2014; 59: 1627-1634.

34. Winston A, Fätkenheuer G, Arribas J, Hill A, van Delft Y, Moecklinghoff $C$. Neuropsychiatric adverse events with ritonavir-boosted darunavir monotherapy in HIV-infected individuals: a randomised prospective study. HIV Clin Trials 2010; 11:163-169.

35. Ellis RJ, Badiee J, Florin V, Scott L, Heaton RK, Clifford D, et al. CD4 nadir is a predictor of HIV neurocognitive impairment in the era of combination antiretroviral therapy. AIDS 2011; 25:1747-1751.

36. Dahl V, Peterson J, Fuchs D, Gisslen M, Palmer S, Price RW. Low levels of HIV-1 RNA detected in the cerebrospinal fluid after up to 10 years of suppressive therapy are associated with local immune activation. AIDS 2014; 28:2251-2258.

37. Paton N, Meynard JL, Pulido F, Arenas-Pinto A, Girard PM, Arribas J. Inappropriate claim of 'failure of ritonavirboosted lopinavir monotherapy in HIV' in the Monotherapy Switzerland/Thailand (MOST) trial. AIDS 2011; 25:393-394.

38. Santos JR, Muñoz-Moreno JA, Moltó J, Prats A, Curran A, Domingo $\mathrm{P}$, et al. Virological efficacy in cerebrospinal fluid and neurocognitive status in patients with long-term monotherapy based on lopinavir/ritonavir: an exploratory study. PLoS One 2013; 8:e70201. 